\title{
PREDICTION OF FLEXURAL STRENGTH OF CHIKOKO POZZOLANA BLENDED CEMENT CONCRETE USING OSADEBE'S REGRESSION FUNCTION
}

\author{
D. O. Onwuka ${ }^{1}$ and S. Sule ${ }^{2 *}$ \\ 1 Department of Civil Engineering, Federal University of TeChnology, OWERri, Imo State. Nigeria

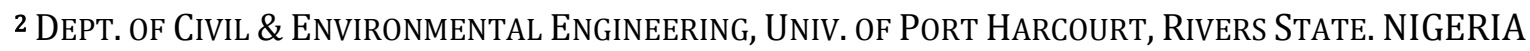 \\ E-mail addresses: ${ }^{1}$ daviesonwuka@yahoo.com, ${ }^{2}$ samvictoryahead@yahoo.com
}

\begin{abstract}
Chikoko mud is abundant in the mangrove swamps of the Niger Delta area of Nigeria. Its utilization in concrete production is traceable to its pozzolanic properties. In this paper, a regression model is developed to predict and optimize the flexural strength of chikoko pozzolana blended cement concrete using Osadebe's regression function. The results obtained from the derived regression model are very close to those obtained from experiment. The model was tested for adequacy using a Fisher test at 5\% level of significance and was found to be adequate. A computer program coded in basic language was used to select the mix ratios that would optimize the flexural strength of chikoko pozzolana blended cement concrete. The computer program is user-friendly and can be used to select the mix ratios corresponding to a desired strength value with reasonable accuracy and without waste of time.
\end{abstract}

Keywords: Pozzolanic properties, Osadebe's regression function, flexural strength, desired strength, mix ratios

\section{INTRODUCTION}

In Nigeria, the provision of decent accommodation at an affordable rate to low income earners has been difficult over the years due to high cost of building materials such as cement [1-3]. Nigeria is blessed with abundance of local building and construction materials such as stones, sand, laterite and timber. However, majority of Nigerians still find it difficult to afford their own shelters due to high cost of cement. Concrete is the most important component of concrete as a structural material in the world [4]. Concrete is a combination of cement, fine and coarse aggregates and water, which are mixed in a particular proportion to achieve a particular strength [5]. The chemical reaction of cement with water forms a paste that binds the aggregates together. The concrete mixture then undergoes a hardening process to form a rock-like material that has high compressive but low tensile strength.

The price of cement is soaring high. Consequently, concrete structures and houses to accommodate the teeming population of Nigerians are difficult to construct [6-9]. There is therefore an immediate need to explore the potentials of locally processed building and construction materials such as chikoko to replace cement partially without negatively affecting the quality and strength properties of concrete [10].These materials are called natural pozzolanas. The use of natural pozzolanas in concrete slows down the hydration process in concrete and results in low rate of heat development in concrete [11-12]. Pozzolanic reaction has been reported to improve concrete impermeability [13]. After a long while, chikoko pozzolana-cement concrete structures may show signs of structural failure due to lack or insufficient knowledge of the predictive models on the structural property such as flexural strength of the end products. The total cost of concrete production depends on the proportions of the component materials. This implies that the addition of chikoko as one of the ingredients would result in increase in the cost per $\mathrm{m}^{3}$ of concrete. Consequently, the formulation of optimization model becomes a necessity in order to select the best mix ratios that would optimize the concrete property of interest at minimum practicable cost [14].

This paper aims at predicting and optimizing the flexural strength of chikoko pozzolana concrete using Osadebe's regression theory and to develop a computer program for easy, quick and accurate prediction of flexural strength.

\section{OSADEBE'SREGRESSION THEORY}

The Osadebe's regression theory is based on the principle of absolute volume. Consider the 5-component 
concrete mixture to have a total quantity, $S$ and the proportion of the ith component material as $S_{i}$.

Osadebe [9] assumed the response function, $f(Z)$ to be continuous and differentiable with respect to its predictors $Z_{i}$ The function $f(Z)$ can be expanded in Taylor's series in the neighborhood of a chosen point $Z^{(0)}$.

$$
Z^{(0)}=Z_{1}^{(0)}, Z_{2}{ }^{(0)}, Z_{3}{ }^{(0)}, Z_{4}{ }^{(0)}, Z_{5}{ }^{(0)}
$$

Osadebe gave the regression function for predicting and optimizing concrete properties as:

$$
\begin{aligned}
& f(Z) \\
& =f\left(Z^{(0)}\right)+\sum_{i=1}^{5} \frac{\partial f\left(Z^{(0)}\right)}{\partial Z_{i}}\left(Z_{i}-Z_{i}^{(0)}\right) \\
& +\frac{1}{2} ! \sum_{i=1}^{4} \sum_{j=1}^{5} \frac{\partial f\left(Z^{(0)}\right)}{\partial Z_{i} \partial Z_{j}}\left(Z_{i}-Z_{i}^{(0)}\right)\left(Z_{j}-Z_{j}^{(0)}\right) \\
& +\frac{1}{2} ! \sum_{i=1}^{5} \frac{\partial^{2} f\left(Z^{(0)}\right)}{\partial Z_{i}^{2}}\left(Z_{i}-Z_{i}^{(0)}\right)^{2} \\
& +\cdots
\end{aligned}
$$

Let $Z_{i}$ and $S_{i}$ represent the volume fraction and actual proportions of the mixture respectively, then:

$$
\begin{aligned}
& \sum_{i=1}^{5} S_{i}=S \\
& \Rightarrow S_{i}+S_{2}+S_{3}+S_{4}+S_{4}=S
\end{aligned}
$$

Dividing both sides of equation (4) by $S$ yields:

$$
\begin{aligned}
& S_{1} / S+S_{2} / S+S_{3} / S+S_{4} / S+S_{5} / S=1 \\
& S_{i} / S=Z_{i}(i=1,2,3,4,5)
\end{aligned}
$$

Equation (5) now becomes:

$$
Z_{1}+Z_{2}+Z_{3}+Z_{4}+Z_{5}=1
$$

Where:

$Z_{1}, Z_{2}, Z_{3}, Z_{4}$ and $Z_{5}$ is the volume fraction of water, cement, chikoko, sand and coarse aggregate respectively. A vector $Z Z_{1}, Z_{2}, Z_{3}, Z_{4}, Z_{5}$ exists whose elements are subject to the constraint of Equation (7). For each $Z_{i}$

$$
Z_{i}>0
$$

Taking the point $Z^{(0)}$ as the origin means that $Z^{(0)}=0$.

$$
\begin{aligned}
\Rightarrow Z_{1}{ }^{(0)} & =0, Z_{2}{ }^{(0)}=0, Z_{3}{ }^{(0)}=0, Z_{4}{ }^{(0)}=0, Z_{5}{ }^{(0)} \\
& =0 \text {. } \quad(9)
\end{aligned}
$$

Lets $b_{0}=f(0), b_{i}=\frac{\partial f(0)}{\partial Z_{i}}, b_{i j}=\frac{\partial^{2} f(0)}{\partial Z_{i} \partial Z_{j}}$, and $b_{i i}=\frac{\partial^{2} f(0)}{\partial Z_{i}{ }^{2}}$, Equation (2) now becomes:

$$
\begin{aligned}
f(z)=b_{0}+\sum_{i=1}^{5} b_{i} z_{i} & \\
& +\sum_{i=1}^{4} \sum_{i=1}^{5} b_{i j} z_{i} z_{j}+\sum_{i=j}^{5} b_{i i} z_{i}^{2}+\ldots
\end{aligned}
$$

The number of constant coefficients of Equation (10) is:

$$
N=\frac{q(q+1)(q+2) \ldots(q+m+1)}{m !}
$$

In (11), $\mathrm{q}$ is the number of components, and $\mathrm{m}$ is the degree of polynomial. Multiplying (7) by $b_{0}$ yields:

$$
b_{0}=b_{0} Z_{1}+b_{0} Z_{2}+b_{0} Z_{3}+b_{0} Z_{4}+b_{0} Z_{5}
$$

Also, multiplying Equation (7) by $Z_{i}$ yields:

$$
\begin{gathered}
Z_{1}=Z_{1}{ }^{2}+Z_{1} Z_{2}+Z_{1} Z_{3}+Z_{1} Z_{4}+Z_{1} Z_{5} \\
Z_{2}=Z_{2}{ }^{2}+Z_{1} Z_{2}+Z_{2} Z_{3}+Z_{2} Z_{4}+Z_{2} Z_{5} \\
Z_{3}=Z_{3}{ }^{2}+Z_{1} Z_{3}+Z_{3} Z_{3}+Z_{3} Z_{4}+Z_{3} Z_{5} \\
Z_{4}=Z_{4}{ }^{2}+Z_{1} Z_{4}+Z_{2} Z_{4}+Z_{4} Z_{3}+Z_{4} Z_{5} \\
Z_{5}=Z_{5}{ }^{2}+Z_{1} Z_{5}+Z_{2} Z_{5}+Z_{3} Z_{5}+Z_{4} Z_{5}
\end{gathered}
$$

Rearranging Equations (13-17), yields:

$$
\begin{gathered}
Z_{1}^{2}=Z_{1}-Z_{1} Z_{2}-Z_{1} Z_{3}-Z_{1} Z_{4}-Z_{1} Z_{5} \\
Z_{2}^{2}=Z_{2}-Z_{1} Z_{2}-Z_{2} Z_{3}-Z_{2} Z_{4}-Z_{2} Z_{5} \\
Z_{3}^{2}=Z_{3}-Z_{1} Z_{3}-Z_{2} Z_{3}-Z_{3} Z_{4}-Z_{3} Z_{5} \\
Z_{4}^{2}=Z_{4}-Z_{1} Z_{1}-Z_{2} Z_{4}-Z_{4} Z_{3}-Z_{4} Z_{5} \\
Z_{5}^{2}=Z_{5}-Z_{1} Z_{5}-Z_{2} Z_{5}-Z_{3} Z_{5}-Z_{4} Z_{5}
\end{gathered}
$$

Substituting Equations (18) to (22) into Equation (10) and setting $\mathrm{f}(0)=\mathrm{y}$ yields:

$y$

$=b_{0} Z_{1}+b_{0} Z_{2}+b_{0} Z_{3}+b_{0} Z_{4}+b_{0} Z_{5}+b_{1} Z_{1}+b_{2} Z_{2}$

$+b_{3} Z_{3}+b_{4} Z_{4}+b_{5} Z_{5}+b_{12} Z_{1} Z_{2}+b_{13} Z_{1} Z_{3}+b_{14} Z_{1} Z_{4}$

$+b_{15} Z_{1} Z_{5}+b_{23} Z_{2} Z_{3}+b_{24} Z_{2} Z_{4}+b_{25} Z_{2} Z_{5}+b_{34} Z_{3} Z_{4}$

$+b_{35} Z_{3} Z_{5}+b_{45} Z_{4} Z_{5}$

$+b_{11}\left(Z_{1}-Z_{1} Z_{2}-Z_{1} Z_{3}-Z_{1} Z_{4}-Z_{1} Z_{5}\right)$

$+b_{22}\left(Z_{2}-Z_{1} Z_{2}-Z_{2} Z_{3}-Z_{2} Z_{4}-Z_{2} Z_{5}\right)$

$+b_{33}\left(Z_{3}-Z_{1} Z_{3}-Z_{2} Z_{3}-Z_{3} Z_{4}-Z_{3} Z_{5}\right)$

$+b_{44}\left(Z_{4}-Z_{1} Z_{4}-Z_{2} Z_{4}-Z_{3} Z_{4}\right.$

$\left.-Z_{4} Z_{5}\right)$

Factorization of Equation (23) yields:

$$
\begin{aligned}
y=\left(b_{0}+b_{1}+\right. & \left.b_{11}\right) Z_{1}+\left(b_{0}+b_{2}+b_{22}\right) Z_{2} \\
& +\left(b_{0}+b_{3}+b_{33}\right) Z_{3} \\
& +\left(b_{0}+b_{4}+b_{44}\right) Z_{4} \\
& +\left(b_{0}+b_{5}+b_{55}\right) Z_{5} \\
& +\left(b_{12}+b_{11}+b_{22}\right) Z_{1} Z_{2} \\
& +\left(b_{13}-b_{11}-b_{33}\right) Z_{1} Z_{3} \\
& +\left(b_{14}-b_{11}-b_{44}\right) Z_{1} Z_{4} \\
& +\left(b_{15}-b_{11}-b_{55}\right) Z_{1} Z_{5} \\
& +\left(b_{23}-b_{22}-b_{33}\right) Z_{2} Z_{3} \\
& +\left(b_{24}-b_{22}-b_{44}\right) Z_{2} Z_{4} \\
& +Z_{2} Z_{5}\left(b_{25}-b_{22}-b_{55}\right) \\
& +\left(b_{34}-b_{33}-b_{44}\right) Z_{3} Z_{4} \\
& +Z_{3} Z_{5}\left(b_{35}-b_{33}+b_{55}\right) \\
& +Z_{4} Z_{5}\left(b_{45}-b_{44}\right. \\
& \left.-b_{55}\right) \\
&
\end{aligned}
$$

Let:

And

$$
\alpha_{i}=b_{0}+b_{i}+b_{i i}
$$

$$
\alpha_{i j}=b_{i j}+b_{i i}+b_{j j}
$$

Substituting Equations (25) and (26) into Equation (24) yields: 


$$
\begin{aligned}
y=\alpha_{1} Z_{1}+\alpha_{2} Z_{2} & +\alpha_{3} Z_{3}+\alpha_{4} Z_{4}+\alpha_{5} Z_{5}+\alpha_{12} Z_{1} Z_{2} \\
& +\alpha_{13} Z_{1} Z_{3}+\alpha_{14} Z_{1} Z_{4}+\alpha_{15} Z_{1} Z_{5} \\
& +\alpha_{23} Z_{2} Z_{3}+\alpha_{24} Z_{2} Z_{4}+\alpha_{25} Z_{2} Z_{5} \\
& +\alpha_{34} Z_{3} Z_{4}+\alpha_{35} Z_{3} Z_{5}+\alpha_{45} Z_{4} Z_{5}
\end{aligned}
$$

Equation (27) is the regression model for predicting the property of a 5-component mixture based on Osadebe's second degree polynomial.

The generalized form of Equation (27) is:

$$
y=\sum_{i=1}^{5} \alpha_{i} Z_{i}+\sum_{1 \leq i \leq j}^{5} \alpha_{i j} Z_{i} Z_{j}
$$

In Equation (28), $\mathrm{Y}$ is the flexural strength at any point of observation, $Z_{i}$ and $Z_{j}$ is the predictor variables and $\alpha_{i j}=\alpha_{i j}$ is the coefficients of the regression model.

\subsection{Coefficients of the Osadebe's Regression Model}

Let $\mathrm{y}^{(\mathrm{n})}$ represent the $\mathrm{n}^{\text {th }}$ point of observation. The vector of the corresponding volume fraction is:

$$
Z^{(n)}=\left[Z_{1}{ }^{(n)}, Z_{2}{ }^{(n)}, Z_{3}{ }^{(n)}, Z_{5}{ }^{(n)},\right]
$$

At $n^{\text {th }}$ observation point, the response function, $y^{(n)}$ corresponds with the predictors, $Z_{i}^{(n)}$.

$$
\Rightarrow y^{(n)}=\sum_{i=1}^{5} \alpha_{i} Z_{i}{ }^{(n)}+\sum_{1 \leq i \leq j}^{5} \alpha_{i j} Z_{i}{ }^{(n)} Z_{j}{ }^{(n)}
$$

Where: $1 \leq i \leq j \leq 5$ and $n=1,2,3 \ldots, 15$

Equation (30) can be written in matrix form as:

$$
\left[y^{(n)}\right]=\left[y^{(n)}\right][\alpha]
$$

Expanding Equation (31), we have:

$$
\left[\begin{array}{c}
y^{(1)} \\
y^{(2)} \\
y^{(3)} \\
\cdot \\
\cdot \\
\cdot \\
y^{(15)}
\end{array}\right]=\left[\begin{array}{ccccc}
Z_{1}^{(1)} & Z_{2}^{(1)} & Z_{3}^{(1)} & \ldots & Z_{4}^{(1)} Z_{5}^{(1)} \\
Z_{1}^{(2)} & Z_{2}^{(2)} & Z_{3}^{(2)} & \ldots & Z_{4}^{(2)} Z_{5}^{(2)} \\
Z_{1}^{(3)} & Z_{2}^{(3)} & Z_{3}^{(3)} & \ldots & Z_{4}^{(3)} Z_{5}^{(3)} \\
& & \cdot & & \\
& & \vdots & & \\
Z_{1}^{(15)} & Z_{2}^{(15)} & Z_{3}^{(15)} & \ldots & Z_{4}^{(15)} Z_{5}^{(15)}
\end{array}\right]\left[\begin{array}{c}
\alpha_{1} \\
\alpha_{2} \\
\alpha_{3} \\
\cdot \\
\cdot \\
\cdot \\
\alpha_{45}
\end{array}\right]
$$

The constant coefficients $\alpha_{i}$ in Equation (31) are determined from the values of $y^{(n)}$ and $Z^{(n)}$. Rearrangement of Equation (32) yields:

$$
[\alpha]=\left[Z^{(n)}\right]^{-1}=\left[y^{(n)}\right]
$$

Expressing (33) in matrix form yields:

$$
\left[\begin{array}{c}
\alpha^{(1)} \\
\alpha^{(2)} \\
\alpha^{(3)} \\
\cdot \\
\cdot \\
\cdot \\
\alpha^{(15)}
\end{array}\right]=\left[\begin{array}{ccccc}
Z_{1}^{(1)} & Z_{2}^{(1)} & Z_{3}^{(1)} & \ldots & Z_{4}^{(1)} Z_{5}^{(1)} \\
Z_{1}^{(2)} & Z_{2}^{(2)} & Z_{3}^{(2)} & \ldots & Z_{4}^{(2)} Z_{5}^{(2)} \\
Z_{1}^{(3)} & Z_{2}^{(3)} & Z_{3}^{(3)} & \ldots & Z_{4}^{(3)} Z_{5}^{(3)} \\
& & \cdot & & \\
& & \cdot & & \\
Z_{1}^{(15)} & Z_{2}^{(15)} & Z_{3}^{(15)} & \ldots & Z_{4}^{(15)} Z_{5}^{(15)}
\end{array}\right]\left[\begin{array}{c}
y^{1} \\
y^{2} \\
y^{2} \\
\cdot \\
\cdot \\
y^{15}
\end{array}\right]
$$

The actual proportions $S_{i}$ and the corresponding volume fractions $Z_{i}^{(n)}$ are given in Table 2. The values of the volume fraction $Z_{i}{ }^{(n)}$ were used to determine $Z^{(n)}$ matrix (Table 3) and $Z^{(n)}$ matrix inverse (Table 4). The values of $y^{(n)}$ matrix were determined from experiments. The values of the constant coefficients $\alpha_{i}$ were determined from known values of the matrices $y^{(n)}$ and $Z^{(n)}$ using Equation (34).

\section{MATERIALS AND METHODS}

The cement used for this study was ordinary Portland cement with properties meeting the requirements of [15].The water used in this study was clean, fresh and free from organic matters. The fine aggregate was obtained from Otamiri River in Owerri, Imo State. It was washed and air-dried for a period of two weeks before using it to produce concrete. The grading and properties were determined and met the requirements of [16]. The maximum size of the fine aggregate did not exceed $5 \mathrm{~mm}$. The granite aggregate used was obtained from crushed rock industry in Port Harcourt, Rivers State. The granites were properly washed and sundried for two weeks to remove dirt and later surface dried before usage. The maximum size of the granite aggregate was $20 \mathrm{~mm}$. The chikoko was obtained in bags from mangrove swamps at Eagle's Island, Port Harcourt, Rivers State. It was sundried for three (3) weeks after which it was ground and sieved with a $212 \mu \mathrm{m}$ sieve to obtain finer particles and was characterized to determine its suitability for use as a pozzolana (Table 1).The ground and sieved chikoko pozzolana is as shown in Plate 1.The actual mix ratios and their corresponding volume fractions are given in Table 2 .

The flexural strength test of the beam was performed using $450 \mathrm{~mm} \times 150 \mathrm{~mm} \times 150 \mathrm{~mm}$ steel moulds. The proportions of the ingredients were weighed and turned over and over with a shovel until a homogenous mix was obtained. Water was then added and the components were mixed until a uniform colour was achieved. The fresh concrete was then compacted into steel moulds with a tamping rod. A total of ninety (90) concrete beams were produced from both the trial and control mixes implying that three test samples were produced per mix ratio. The beam specimens were removed from moulds after 24 hours of casting and were cured in a curing tank for 28 days and tested for flexural strengths on a Universal Testing Machine using a third point loading method. The test results are as shown in Table 4. Concrete beams fractured in the tension zone within the middle third of the beam span. The flexural strength was determined using the formula:

$$
\sigma=P L / b d^{2}
$$

In Equation (35), $\sigma$ is the flexural strength of the beam specimen, $P$ is the failure load of the beam specimen, $L$ $=$ is the beam span and $b, d$ is the width and depth of the beam respectively. 
Table 1: Characterization of chikoko from experiment

\begin{tabular}{ccc}
\hline S/No & Component & Content (\%) \\
\hline 1 & $\mathrm{CaO}$ & 9.85 \\
2 & $\mathrm{SiO}_{2}$ & 41.21 \\
3 & $\mathrm{Al}_{2} \mathrm{O}_{3}$ & 10.15 \\
4 & $\mathrm{Fe}_{2} \mathrm{O}_{3}$ & 2.31 \\
5 & $\mathrm{MgO}$ & 5.02 \\
6 & $\mathrm{Na}_{2} \mathrm{O}$ & 1.97 \\
7 & $\mathrm{~K}_{2} \mathrm{O}$ & 8.17 \\
8 & $\mathrm{SO}_{3}$ & 0.08 \\
9 & $\mathrm{TiO}_{2}$ & 0.72 \\
10 & $\mathrm{ZnO}$ & 0.09 \\
11 & $\mathrm{LoI}$ & 6.51 \\
\hline
\end{tabular}

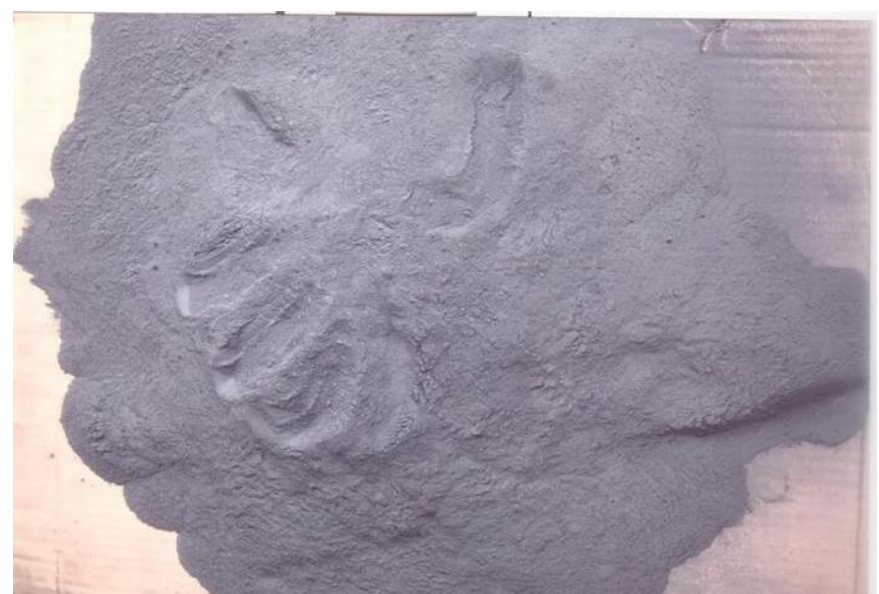

Plate 1: Chikoko

Table 2: Values of actual mix ratios and component fractions based on Osadebe's second degree polynomial

\begin{tabular}{|c|c|c|c|c|c|c|c|c|c|c|}
\hline \multirow{2}{*}{$\mathrm{S} / \mathrm{N}$} & \multicolumn{5}{|c|}{ Actual Mix Ratios } & \multicolumn{5}{|c|}{ Component's Volume Fraction } \\
\hline & S1 & S2 & S3 & S4 & S5 & $\mathrm{Z1}$ & Z2 & $\mathrm{Z3}$ & Z4 & Z5 \\
\hline 1 & 0.52601 & 0.947 & 0.053 & 2.1 & 4.2 & 0.06721305 & 0.121006745 & 0.006772289 & 0.268335972 & 0.536671944 \\
\hline 2 & 0.566 & 0.91901 & 0.081 & 2.02 & 4.04 & 0.074219677 & 0.120509939 & 0.010621544 & 0.264882947 & 0.529765893 \\
\hline 3 & 0.589 & 0.823 & 0.17701 & 1.91 & 3.82 & 0.080475365 & 0.112446902 & 0.024184965 & 0.260964256 & 0.521928512 \\
\hline 4 & 0.611 & 0.889 & 0.111 & 2.1601 & 4.32 & 0.075515072 & 0.109873812 & 0.013718777 & 0.266972352 & 0.533919986 \\
\hline 5 & 0.596 & 0.846 & 0.154 & 2.15 & 4.301 & 0.074064869 & 0.105132347 & 0.019137567 & 0.267180316 & 0.534484901 \\
\hline 6 & 0.546005 & 0.933005 & 0.067 & 2.06 & 4.12 & 0.070671019 & 0.120761557 & 0.008672005 & 0.266631806 & 0.533263612 \\
\hline 7 & 0.557505 & 0.885 & 0.115005 & 2.005 & 4.01 & 0.07362222 & 0.1168701 & 0.01518717 & 0.264773503 & 0.529547006 \\
\hline 8 & 0.568505 & 0.918 & 0.082 & 2.13005 & 4.26 & 0.071433194 & 0.115347573 & 0.010303378 & 0.267642807 & 0.535273049 \\
\hline 9 & 0.561005 & 0.8965 & 0.1035 & 2.125 & 4.2505 & 0.070686656 & 0.112959042 & 0.013041005 & 0.267750099 & 0.535563198 \\
\hline 10 & 0.5775 & 0.871005 & 0.129005 & 1.965 & 3.93 & 0.077283269 & 0.116561236 & 0.017263945 & 0.26296385 & 0.5259277 \\
\hline 11 & 0.5885 & 0.904005 & 0.096 & 2.09005 & 4.18 & 0.074886541 & 0.115034507 & 0.012215986 & 0.265958564 & 0.531904402 \\
\hline 12 & 0.581 & 0.882505 & 0.1175 & 2.085 & 4.1705 & 0.074140194 & 0.112614616 & 0.014993929 & 0.266062486 & 0.532188775 \\
\hline 13 & 0.6 & 0.856 & 0.144005 & 2.03505 & 4.07 & 0.077870956 & 0.111095897 & 0.018689678 & 0.264118816 & 0.528224653 \\
\hline 14 & 0.5925 & 0.8345 & 0.165505 & 2.03 & 4.0605 & 0.077118263 & 0.108616355 & 0.021541701 & 0.264219534 & 0.528504146 \\
\hline 15 & 0.6035 & 0.8675 & 0.1325 & 2.15505 & 4.3105 & 0.074791952 & 0.107509558 & 0.016420768 & 0.26707605 & 0.534201672 \\
\hline \multicolumn{11}{|c|}{ Control Points } \\
\hline 16 & 0.560336667 & 0.896336667 & 0.10367 & 2.01 & 4.02 & 0.073822308 & 0.118089081 & 0.013658144 & 0.264810156 & 0.529620311 \\
\hline 17 & 0.575336667 & 0.886333333 & 0.11367 & 2.0567 & 4.113333333 & 0.074281334 & 0.114433907 & 0.014675858 & 0.265539169 & 0.531069731 \\
\hline 18 & 0.57767 & 0.894 & 0.106 & 2.1367 & 4.2736666667 & 0.072316894 & 0.111917363 & 0.013269844 & 0.267487505 & 0.535008394 \\
\hline 19 & 0.5730025 & 0.8945025 & 0.1055025 & 2.047525 & 4.095 & 0.074266099 & 0.115935290 & 0.013674040 & 0.265377017 & 0.530747554 \\
\hline 20 & 0.5805025 & 0.87625 & 0.1237525 & 2.080025 & 4.16025 & 0.074225653 & 0.112041254 & 0.015823550 & 0.265961324 & 0.531948220 \\
\hline 21 & 0.5692525 & 0.8837525 & 0.1162525 & 2.045 & 4.09025 & 0.073885644 & 0.114705904 & 0.015088894 & 0.265429036 & 0.530890521 \\
\hline 22 & 0.551755 & 0.9090025 & 0.0910025 & 2.0325 & 4.065 & 0.072131814 & 0.118835351 & 0.011896902 & 0.265711977 & 0.531423955 \\
\hline 23 & 0.5767525 & 0.8655 & 0.1345025 & 2.0775 & 4.1555 & 0.073850268 & 0.110822939 & 0.017222371 & 0.266013466 & 0.532090955 \\
\hline 24 & 0.563604 & 0.905002 & 0.095002 & 2.05802 & 4.116 & 0.072839377 & 0.116961167 & 0.012277923 & 0.265975568 & 0.531945966 \\
\hline 25 & 0.577602 & 0.884802 & 0.115202 & 2.06802 & 4.1362 & 0.074224482 & 0.113701077 & 0.014803980 & 0.265749967 & 0.531520494 \\
\hline 26 & 0.573603 & 0.887601 & 0.112402 & 2.07602 & 4.1522 & 0.073521635 & 0.113768367 & 0.014407140 & 0.266094117 & 0.532208742 \\
\hline 27 & 0.586101 & 0.879002 & 0.121002 & 2.07403 & 4.1482 & 0.075060944 & 0.112572271 & 0.015496518 & 0.265617446 & 0.531252822 \\
\hline 28 & 0.5652535 & 0.8865515 & 0.1134525 & 2.053 & 4.10625 & 0.073176639 & 0.114771265 & 0.014687344 & 0.265777462 & 0.531587289 \\
\hline 29 & 0.5744525 & 0.891002 & 0.1090015 & 2.07752 & 4.1552 & 0.073580063 & 0.114126030 & 0.013961707 & 0.266103902 & 0.532228299 \\
\hline
\end{tabular}




\begin{tabular}{|c|c|c|c|c|c|c|c|c|c|c|}
\hline \multirow{2}{*}{ S/N } & \multicolumn{4}{|c|}{ Actual Mix Ratios } & \multicolumn{4}{c|}{ Component's Volume Fraction } \\
\cline { 2 - 11 } & S1 & S2 & S3 & S4 & S5 & Z1 & Z2 & Z3 & Z4 & Z5 \\
\hline 30 & 0.5660045 & 0.9037005 & 0.0963 & 2.12302 & 4.2463 & 0.071327198 & 0.113883237 & 0.012135609 & 0.267540397 & 0.535113559 \\
\hline
\end{tabular}

Table 3: Znmatrix obtained from Table 2

\begin{tabular}{|c|c|c|c|c|c|c|c|c|c|c|c|c|c|c|}
\hline \multicolumn{15}{|c|}{ Zn MATRIX } \\
\hline $\mathrm{Z1}$ & Z2 & Z3 & Z4 & Z5 & Z1Z2 & Z1Z3 & Z1Z4 & Z1Z5 & Z2Z3 & Z2Z4 & Z2Z5 & Z3Z4 & Z3Z5 & Z4ZZ \\
\hline $\begin{array}{l}0.06721 \\
305\end{array}$ & $\begin{array}{l}0.12100 \\
6745\end{array}$ & $\begin{array}{l}0.00677 \\
2289\end{array}$ & $\begin{array}{l}0.26833 \\
5972\end{array}$ & $\begin{array}{l}0.53667 \\
1944\end{array}$ & $\begin{array}{l}0.00813 \\
3232\end{array}$ & $\begin{array}{l}0.00045 \\
5186\end{array}$ & $\begin{array}{l}0.01803 \\
5679\end{array}$ & $\begin{array}{l}0.03607 \\
1358\end{array}$ & $\begin{array}{l}0.00081 \\
9493\end{array}$ & $\begin{array}{l}0.03247 \\
0463\end{array}$ & $\begin{array}{l}0.06494 \\
0925\end{array}$ & $\begin{array}{l}0.00181 \\
7249\end{array}$ & $\begin{array}{l}0.00363 \\
4497\end{array}$ & $\begin{array}{l}0.14400 \\
8388\end{array}$ \\
\hline $\begin{array}{l}0.07421 \\
9677\end{array}$ & $\begin{array}{l}0.12050 \\
9939\end{array}$ & $\begin{array}{l}0.01062 \\
1544\end{array}$ & $\begin{array}{l}0.26488 \\
2947\end{array}$ & $\begin{array}{l}0.52976 \\
5893\end{array}$ & $\begin{array}{l}0.00894 \\
4209\end{array}$ & $\begin{array}{l}0.00078 \\
8328\end{array}$ & $\begin{array}{l}0.01965 \\
9527\end{array}$ & $\begin{array}{l}0.03931 \\
9054\end{array}$ & $\begin{array}{l}0.00128 \\
0002\end{array}$ & $\begin{array}{l}0.03192 \\
1028\end{array}$ & $\begin{array}{l}0.06384 \\
2055\end{array}$ & $\begin{array}{l}0.00281 \\
3466\end{array}$ & $\begin{array}{l}0.00562 \\
6932\end{array}$ & $\begin{array}{l}0.14032 \\
5951\end{array}$ \\
\hline $\begin{array}{l}0.08047 \\
5365\end{array}$ & $\begin{array}{l}0.11244 \\
6902\end{array}$ & $\begin{array}{l}0.02418 \\
4965\end{array}$ & $\begin{array}{l}0.26096 \\
4256\end{array}$ & $\begin{array}{l}0.52192 \\
8512\end{array}$ & $\begin{array}{l}0.00904 \\
9205\end{array}$ & $\begin{array}{l}0.00194 \\
6294\end{array}$ & $\begin{array}{l}0.02100 \\
1194\end{array}$ & $\begin{array}{l}0.04200 \\
2387\end{array}$ & $\begin{array}{l}0.00271 \\
9524\end{array}$ & $\begin{array}{l}0.02934 \\
4622\end{array}$ & $\begin{array}{l}0.05868 \\
9244\end{array}$ & $\begin{array}{l}0.00631 \\
1411\end{array}$ & $\begin{array}{l}0.01262 \\
2823\end{array}$ & $\begin{array}{l}0.13620 \\
4686\end{array}$ \\
\hline $\begin{array}{l}0.07551 \\
5072\end{array}$ & $\begin{array}{l}0.10987 \\
3812\end{array}$ & $\begin{array}{l}0.01371 \\
8777\end{array}$ & $\begin{array}{l}0.26697 \\
2352\end{array}$ & $\begin{array}{l}0.53391 \\
9986\end{array}$ & $\begin{array}{l}0.00829 \\
7129\end{array}$ & $\begin{array}{l}0.00103 \\
5974\end{array}$ & $\begin{array}{l}0.02016 \\
0436\end{array}$ & $\begin{array}{l}0.04031 \\
9006\end{array}$ & $\begin{array}{l}0.00150 \\
7334\end{array}$ & $\begin{array}{l}0.02933 \\
327\end{array}$ & $\begin{array}{l}0.05866 \\
3824\end{array}$ & $\begin{array}{l}0.00366 \\
2534\end{array}$ & $\begin{array}{l}0.00732 \\
4729\end{array}$ & $\begin{array}{l}0.14254 \\
1875\end{array}$ \\
\hline $\begin{array}{l}0.07406 \\
4869\end{array}$ & $\begin{array}{l}0.10513 \\
2347\end{array}$ & $\begin{array}{l}0.01913 \\
7567\end{array}$ & $\begin{array}{l}0.26718 \\
0316\end{array}$ & $\begin{array}{l}0.53448 \\
4901\end{array}$ & $\begin{array}{l}0.00778 \\
6614\end{array}$ & $\begin{array}{l}0.00141 \\
7421\end{array}$ & $\begin{array}{l}0.01978 \\
8675\end{array}$ & $\begin{array}{l}0.03958 \\
6554\end{array}$ & $\begin{array}{l}0.00201 \\
1977\end{array}$ & $\begin{array}{l}0.02808 \\
9294\end{array}$ & $\begin{array}{l}0.05619 \\
1652\end{array}$ & $\begin{array}{l}0.00511 \\
3181\end{array}$ & $\begin{array}{l}0.01022 \\
874\end{array}$ & $\begin{array}{l}0.14280 \\
3845\end{array}$ \\
\hline $\begin{array}{l}0.07067 \\
1019\end{array}$ & $\begin{array}{l}0.12076 \\
1557\end{array}$ & $\begin{array}{l}0.00867 \\
2005\end{array}$ & $\begin{array}{l}0.26663 \\
1806\end{array}$ & $\begin{array}{l}0.53326 \\
3612\end{array}$ & $\begin{array}{l}0.00853 \\
4342\end{array}$ & $\begin{array}{l}0.00061 \\
2859\end{array}$ & & $\begin{array}{l}0.03768 \\
6283\end{array}$ & $\begin{array}{l}0.00104 \\
7245\end{array}$ & $\begin{array}{l}0.03219 \\
8872\end{array}$ & $\begin{array}{l}0.06439 \\
7744\end{array}$ & $\begin{array}{l}0.00231 \\
2232\end{array}$ & $\begin{array}{l}0.00462 \\
4465\end{array}$ & $\begin{array}{l}0.14218 \\
504\end{array}$ \\
\hline $\begin{array}{l}0.07362 \\
222\end{array}$ & $\begin{array}{l}0.11687 \\
01\end{array}$ & $\begin{array}{l}0.01518 \\
717\end{array}$ & $\begin{array}{l}0.26477 \\
3503\end{array}$ & $\begin{array}{l}0.52954 \\
7006\end{array}$ & $\begin{array}{l}0.00860 \\
4236\end{array}$ & $\begin{array}{l}0.00111 \\
8113\end{array}$ & $\begin{array}{l}0.01949 \\
3213\end{array}$ & $\begin{array}{l}0.03898 \\
6426\end{array}$ & $\begin{array}{l}0.00177 \\
4926\end{array}$ & $\begin{array}{l}0.03094 \\
4106\end{array}$ & $\begin{array}{l}0.06188 \\
8212\end{array}$ & $\begin{array}{l}0.00402 \\
116\end{array}$ & $\begin{array}{l}0.00804 \\
2321\end{array}$ & $\begin{array}{l}0.14021 \\
0016\end{array}$ \\
\hline $\begin{array}{l}0.07143 \\
3194\end{array}$ & $\begin{array}{l}0.11534 \\
7573\end{array}$ & $\begin{array}{l}0.01030 \\
3378\end{array}$ & $\begin{array}{l}0.26764 \\
2807\end{array}$ & $\begin{array}{l}0.53527 \\
3049\end{array}$ & $\begin{array}{l}0.00823 \\
9645\end{array}$ & $\begin{array}{l}0.00073 \\
6003\end{array}$ & $\begin{array}{l}0.01911 \\
858\end{array}$ & $\begin{array}{l}0.03823 \\
6263\end{array}$ & $\begin{array}{l}0.00118 \\
847\end{array}$ & $\begin{array}{l}0.03087 \\
1948\end{array}$ & $\begin{array}{l}0.06174 \\
2447\end{array}$ & $\begin{array}{l}0.00275 \\
7625\end{array}$ & $\begin{array}{l}0.00551 \\
5121\end{array}$ & $\begin{array}{l}0.14326 \\
1981\end{array}$ \\
\hline $\begin{array}{l}0.07068 \\
6656 \\
\end{array}$ & $\begin{array}{l}0.11295 \\
9042 \\
\end{array}$ & $\begin{array}{l}0.01304 \\
1005 \\
\end{array}$ & $\begin{array}{l}0.26775 \\
0099\end{array}$ & $\begin{array}{l}0.53556 \\
3198 \\
\end{array}$ & $\begin{array}{l}0.00798 \\
4697 \\
\end{array}$ & $\begin{array}{l}0.00092 \\
1825 \\
\end{array}$ & $\begin{array}{l}0.01892 \\
6359 \\
\end{array}$ & $\begin{array}{l}0.03785 \\
7172 \\
\end{array}$ & $\begin{array}{l}0.00147 \\
3099 \\
\end{array}$ & $\begin{array}{l}0.03024 \\
4795 \\
\end{array}$ & $\begin{array}{l}0.06049 \\
6706 \\
\end{array}$ & $\begin{array}{l}0.00349 \\
173 \\
\end{array}$ & $\begin{array}{l}0.00698 \\
4282 \\
\end{array}$ & $\begin{array}{l}0.14339 \\
7099 \\
\end{array}$ \\
\hline $\begin{array}{l}0.07728 \\
3269 \\
\end{array}$ & $\begin{array}{l}0.11656 \\
1236 \\
\end{array}$ & $\begin{array}{l}0.01726 \\
3945 \\
\end{array}$ & $\begin{array}{l}0.26296 \\
385 \\
\end{array}$ & $\begin{array}{l}0.52592 \\
77 \\
\end{array}$ & $\begin{array}{l}0.00900 \\
8233\end{array}$ & $\begin{array}{l}0.00133 \\
4214 \\
\end{array}$ & $\begin{array}{l}0.02032 \\
2706 \\
\end{array}$ & $\begin{array}{l}0.04064 \\
5412 \\
\end{array}$ & $\begin{array}{l}0.00201 \\
2307 \\
\end{array}$ & $\begin{array}{l}0.03065 \\
1391 \\
\end{array}$ & $\begin{array}{l}0.06130 \\
2783 \\
\end{array}$ & $\begin{array}{l}0.00453 \\
9793 \\
\end{array}$ & $\begin{array}{l}0.00907 \\
9587\end{array}$ & $\begin{array}{l}0.13829 \\
9973 \\
\end{array}$ \\
\hline $\begin{array}{l}0.07488 \\
6541\end{array}$ & $\begin{array}{l}0.11503 \\
4507\end{array}$ & $\begin{array}{l}0.01221 \\
5986\end{array}$ & $\begin{array}{l}0.26595 \\
8564\end{array}$ & $\begin{array}{l}0.53190 \\
4402\end{array}$ & $\begin{array}{l}0.00861 \\
4536\end{array}$ & $\begin{array}{l}0.00091 \\
4813 \\
\end{array}$ & $\begin{array}{l}0.01991 \\
6717\end{array}$ & $\begin{array}{l}0.03983 \\
2481\end{array}$ & $\begin{array}{l}0.00140 \\
526\end{array}$ & $\begin{array}{l}0.03059 \\
4412\end{array}$ & $\begin{array}{l}0.06118 \\
7361\end{array}$ & $\begin{array}{l}0.00324 \\
8946\end{array}$ & $\begin{array}{l}0.00649 \\
7737\end{array}$ & $\begin{array}{l}0.14146 \\
4531\end{array}$ \\
\hline $\begin{array}{l}0.07414 \\
0194\end{array}$ & $\begin{array}{l}0.11261 \\
4616\end{array}$ & $\begin{array}{l}0.01499 \\
3929\end{array}$ & $\begin{array}{l}0.26606 \\
2486\end{array}$ & $\begin{array}{l}0.53218 \\
8775\end{array}$ & $\begin{array}{l}0.00834 \\
9269\end{array}$ & $\begin{array}{l}0.00111 \\
1653\end{array}$ & $\begin{array}{l}0.01972 \\
5924\end{array}$ & $\begin{array}{l}0.03945 \\
6579\end{array}$ & $\begin{array}{l}0.00168 \\
8536\end{array}$ & $\begin{array}{l}0.02996 \\
2525\end{array}$ & $\begin{array}{l}0.05993 \\
2234\end{array}$ & $\begin{array}{l}0.00398 \\
9322\end{array}$ & $\begin{array}{l}0.00797 \\
9601\end{array}$ & $\begin{array}{l}0.14159 \\
5469\end{array}$ \\
\hline $\begin{array}{l}0.07787 \\
0956\end{array}$ & $\begin{array}{l}0.11109 \\
5897\end{array}$ & $\begin{array}{l}0.01868 \\
9678\end{array}$ & $\begin{array}{l}0.26411 \\
8816\end{array}$ & $\begin{array}{l}0.52822 \\
4653\end{array}$ & $\begin{array}{l}0.00865 \\
1144\end{array}$ & $\begin{array}{l}0.00145 \\
5383\end{array}$ & $\begin{array}{l}0.02056 \\
7185\end{array}$ & $\begin{array}{l}0.04113 \\
3359\end{array}$ & $\begin{array}{l}0.00207 \\
6347\end{array}$ & $\begin{array}{l}0.02934 \\
2517\end{array}$ & $\begin{array}{l}0.05868 \\
3592\end{array}$ & $\begin{array}{l}0.00493 \\
6296\end{array}$ & $\begin{array}{l}0.00987 \\
2349\end{array}$ & $\begin{array}{l}0.13951 \\
407\end{array}$ \\
\hline $\begin{array}{l}0.07711 \\
8263\end{array}$ & $\begin{array}{l}0.10861 \\
6355\end{array}$ & $\begin{array}{l}0.02154 \\
1701\end{array}$ & $\begin{array}{l}0.26421 \\
9534\end{array}$ & $\begin{array}{l}0.52850 \\
4146\end{array}$ & $\begin{array}{l}0.00837 \\
6305\end{array}$ & $\begin{array}{l}0.00166 \\
1259\end{array}$ & $\begin{array}{l}0.02037 \\
6151\end{array}$ & $\begin{array}{l}0.04075 \\
7322\end{array}$ & $\begin{array}{l}0.00233 \\
9781\end{array}$ & $\begin{array}{l}0.02869 \\
8563\end{array}$ & $\begin{array}{l}0.05740 \\
4194\end{array}$ & $\begin{array}{l}0.00569 \\
1738\end{array}$ & $\begin{array}{l}0.01138 \\
4879\end{array}$ & $\begin{array}{l}0.13964 \\
1119\end{array}$ \\
\hline $\begin{array}{l}0.07479 \\
1952\end{array}$ & $\begin{array}{l}0.10750 \\
9558\end{array}$ & $\begin{array}{l}0.01642 \\
0768\end{array}$ & $\begin{array}{l}0.26707 \\
605\end{array}$ & $\begin{array}{l}0.53420 \\
1672\end{array}$ & $\begin{array}{l}0.00804 \\
085\end{array}$ & $\begin{array}{l}0.00122 \\
8141\end{array}$ & $\begin{array}{l}0.01997 \\
5139\end{array}$ & $\begin{array}{l}0.03995 \\
3986\end{array}$ & $\begin{array}{l}0.00176 \\
539\end{array}$ & $\begin{array}{l}0.02871 \\
3228\end{array}$ & $\begin{array}{l}0.05743 \\
1786\end{array}$ & $\begin{array}{l}0.00438 \\
5594\end{array}$ & $\begin{array}{l}0.00877 \\
2002\end{array}$ & $\begin{array}{l}0.14267 \\
2472\end{array}$ \\
\hline
\end{tabular}

Table 4: Inverse of $Z^{n}$ Matrix Based on Osadebe's Second Degree Polynomial

\begin{tabular}{|c|c|c|c|c|c|c|c|c|c|c|c|c|c|c|}
\hline$Z_{1}$ & $Z_{2}$ & $Z_{3}$ & $Z_{4}$ & $Z_{5}$ & $Z_{1} Z_{2}$ & $Z_{1} Z_{3}$ & $Z_{1} Z_{4}$ & $Z_{1} Z_{5}$ & $Z_{2} Z_{3}$ & $\mathrm{Z}_{2} \mathrm{Z}_{4}$ & $Z_{2} Z_{5}$ & $Z_{3} Z_{4}$ & $Z_{3} Z_{5}$ & $Z_{4} Z_{5}$ \\
\hline $\begin{array}{l}68861 . \\
88734\end{array}$ & $\begin{array}{l}83391 . \\
8\end{array}$ & $\begin{array}{l}9356.8 \\
30938\end{array}$ & $\begin{array}{l}3585.2 \\
75594\end{array}$ & $\begin{array}{l}141.86 \\
30848\end{array}$ & $\begin{array}{l}151583 \\
.542\end{array}$ & $\begin{array}{l}50824 . \\
56129\end{array}$ & $\begin{array}{l}31433 . \\
89793\end{array}$ & $\begin{array}{l}- \\
6251.9 \\
9463 \\
\end{array}$ & $\begin{array}{l}55890 . \\
59649 \\
\end{array}$ & $\begin{array}{l}34612 . \\
02133\end{array}$ & $\begin{array}{l}6883.6 \\
17923 \\
\end{array}$ & $\begin{array}{l}11613 . \\
02714\end{array}$ & $\begin{array}{l}2309.3 \\
33517 \\
\end{array}$ & $\begin{array}{l}1426.2 \\
76382 \\
\end{array}$ \\
\hline $\begin{array}{l}1417.5 \\
37624\end{array}$ & $\begin{array}{l}2467.2 \\
10281\end{array}$ & $\begin{array}{l}2531.7 \\
76741\end{array}$ & $\begin{array}{l}15219 . \\
10693\end{array}$ & $\begin{array}{l}711.27 \\
66514\end{array}$ & $\begin{array}{l}4250.2 \\
84477\end{array}$ & $\begin{array}{l}4297.3 \\
82545\end{array}$ & $\begin{array}{l}9770.7 \\
58848\end{array}$ & $\begin{array}{l}1943.3 \\
37219\end{array}$ & $\begin{array}{l}5557.9 \\
16002\end{array}$ & $\begin{array}{l}12653 . \\
17904\end{array}$ & $\begin{array}{l}2516.4 \\
56702\end{array}$ & $\begin{array}{l}12802 . \\
14894\end{array}$ & $\begin{array}{l}2545.7 \\
95133\end{array}$ & $\begin{array}{l}5780.1 \\
84632\end{array}$ \\
\hline $\begin{array}{l}24460 . \\
8499\end{array}$ & $\begin{array}{l}55223 . \\
9781\end{array}$ & $\begin{array}{l}31172 . \\
00764\end{array}$ & $\begin{array}{l}8114.4 \\
37185\end{array}$ & $\begin{array}{l}400.06 \\
77064\end{array}$ & $\begin{array}{l}73978 . \\
00843\end{array}$ & $\begin{array}{l}57106 \\
22897\end{array}$ & $\begin{array}{l}27791 \\
28067\end{array}$ & $\begin{array}{l}5527.5 \\
01822\end{array}$ & $\begin{array}{l}83280 . \\
21351\end{array}$ & $\begin{array}{l}40581 . \\
72919\end{array}$ & $\begin{array}{l}8070.8 \\
67617\end{array}$ & $\begin{array}{l}31347 . \\
89137\end{array}$ & $\begin{array}{l}6233.7 \\
4759 \\
\end{array}$ & $\begin{array}{l}3029.4 \\
77063\end{array}$ \\
\hline $\begin{array}{l}204318 \\
116\end{array}$ & $\begin{array}{l}686446 \\
898\end{array}$ & $\begin{array}{l}132899 \\
063.7\end{array}$ & $\begin{array}{l}372509 \\
686.6\end{array}$ & $\begin{array}{l}358016 \\
334\end{array}$ & $\begin{array}{l}749135 \\
171.9\end{array}$ & $\begin{array}{l}329939 \\
076.5\end{array}$ & $\begin{array}{l}551918 \\
114.2\end{array}$ & $\begin{array}{l}541027 \\
436.3\end{array}$ & $\begin{array}{l}604335 \\
940.7\end{array}$ & $\begin{array}{l}- \\
101223 \\
8772\end{array}$ & $\begin{array}{l}992194 \\
695.5\end{array}$ & $\begin{array}{l}446121 \\
802.1\end{array}$ & $\begin{array}{l}- \\
437238 \\
796.4\end{array}$ & $\begin{array}{l}- \\
730387 \\
668.7\end{array}$ \\
\hline $\begin{array}{l}507319 \\
86.81\end{array}$ & $\begin{array}{l}170433 \\
209.4\end{array}$ & $\begin{array}{l}332488 \\
63.52\end{array}$ & $\begin{array}{l}927267 \\
76.03 \\
\end{array}$ & $\begin{array}{l}895823 \\
09.62\end{array}$ & $\begin{array}{l}186003 \\
587.8\end{array}$ & $\begin{array}{l}822334 \\
82.32\end{array}$ & $\begin{array}{l}137213 \\
212.8\end{array}$ & $\begin{array}{l}134854 \\
827.1\end{array}$ & $\begin{array}{l}150618 \\
908.4\end{array}$ & $\begin{array}{l}251646 \\
212.7\end{array}$ & $\begin{array}{l}247303 \\
494.7 \\
\end{array}$ & $\begin{array}{l}111330 \\
631.3\end{array}$ & $\begin{array}{l}109397 \\
106.7\end{array}$ & $\begin{array}{l}182283 \\
322.8\end{array}$ \\
\hline $\begin{array}{l}90092 . \\
69381\end{array}$ & $\begin{array}{l}- \\
57128 . \\
52872\end{array}$ & $\begin{array}{l}- \\
21635 . \\
82023\end{array}$ & $\begin{array}{l}- \\
33581 . \\
93103\end{array}$ & $\begin{array}{l}- \\
1492.0 \\
45384\end{array}$ & $\begin{array}{l}143211 \\
.6885\end{array}$ & $\begin{array}{l}90801 . \\
39295\end{array}$ & $\begin{array}{l}109338 \\
.2428\end{array}$ & $\begin{array}{l}21746 . \\
64636\end{array}$ & $\begin{array}{l}70789 . \\
29429\end{array}$ & $\begin{array}{l}85351 . \\
42743\end{array}$ & $\begin{array}{l}16974 . \\
63195\end{array}$ & $\begin{array}{l}54152 . \\
90492\end{array}$ & $\begin{array}{l}10768 . \\
68321\end{array}$ & $\begin{array}{l}- \\
12948 . \\
98849\end{array}$ \\
\hline $\begin{array}{l}- \\
175418 \\
.9591 \\
\end{array}$ & $\begin{array}{l}- \\
274347 \\
.0075 \\
\end{array}$ & $\begin{array}{l}- \\
74689 . \\
2729 \\
\end{array}$ & $\begin{array}{l}- \\
22492 . \\
63951 \\
\end{array}$ & $\begin{array}{l}- \\
1023.1 \\
88948 \\
\end{array}$ & $\begin{array}{l}439465 \\
.4863 \\
\end{array}$ & $\begin{array}{l}232898 \\
.6329 \\
\end{array}$ & $\begin{array}{l}124106 \\
.2024 \\
\end{array}$ & $\begin{array}{l}24683 . \\
90237\end{array}$ & $\begin{array}{l}287653 \\
.3305 \\
\end{array}$ & $\begin{array}{l}153482 \\
.7544 \\
\end{array}$ & $\begin{array}{l}30524 . \\
55231\end{array}$ & $\begin{array}{l}81395 . \\
17737 \\
\end{array}$ & $\begin{array}{l}16186 . \\
00438\end{array}$ & $\begin{array}{l} \\
8613.0 \\
95675 \\
\end{array}$ \\
\hline $\begin{array}{l}211888 \\
906.1\end{array}$ & $\begin{array}{l}701662 \\
180.3\end{array}$ & $\begin{array}{l}135138 \\
679.8\end{array}$ & $\begin{array}{l}370201 \\
952.5\end{array}$ & $\begin{array}{l}358467 \\
202.7 \\
\end{array}$ & $\begin{array}{l}771296 \\
593.9\end{array}$ & $\begin{array}{l}338815 \\
525\end{array}$ & $\begin{array}{l}560306 \\
802.8\end{array}$ & $\begin{array}{l}551306 \\
658.3\end{array}$ & $\begin{array}{l}616123 \\
628.1\end{array}$ & $\begin{array}{l}102022 \\
0648\end{array}$ & $\begin{array}{l}100376 \\
1982\end{array}$ & $\begin{array}{l}448469 \\
497.9\end{array}$ & $\begin{array}{l}441185 \\
117.1\end{array}$ & $\begin{array}{l}728580 \\
084\end{array}$ \\
\hline $\begin{array}{l}- \\
470626 \\
67.06 \\
\end{array}$ & $\begin{array}{l} \\
162976 \\
678.5 \\
\end{array}$ & $\begin{array}{l}- \\
321426 \\
86.42 \\
\end{array}$ & $\begin{array}{l} \\
938835 \\
32.43 \\
\end{array}$ & $\begin{array}{l}- \\
893569 \\
89.58 \\
\end{array}$ & $\begin{array}{l}175187 \\
973.1\end{array}$ & $\begin{array}{l}- \\
778751 \\
14.03 \\
\end{array}$ & $\begin{array}{l} \\
132979 \\
709.6\end{array}$ & $\begin{array}{l}129723 \\
004.6 \\
\end{array}$ & $\begin{array}{l}144816 \\
413.9 \\
\end{array}$ & $\begin{array}{l}247609 \\
968.4 \\
\end{array}$ & $\begin{array}{l}- \\
241528 \\
862 \\
\end{array}$ & $\begin{array}{l}110143 \\
645.2 \\
\end{array}$ & $\begin{array}{l}107426 \\
559 \\
\end{array}$ & $\begin{array}{l}183185 \\
965.7 \\
\end{array}$ \\
\hline $\begin{array}{l}- \\
14083 . \\
13954\end{array}$ & $\begin{array}{l}- \\
81086 . \\
79542 \\
\end{array}$ & $\begin{array}{l}- \\
15924 . \\
33948\end{array}$ & $\begin{array}{l} \\
1107.1 \\
15161\end{array}$ & $\begin{array}{l} \\
43.799 \\
80248 \\
\end{array}$ & $\begin{array}{l}67597 . \\
15573 \\
\end{array}$ & $\begin{array}{l}- \\
29984 . \\
72891\end{array}$ & $\begin{array}{l}7899.5 \\
66103 \\
\end{array}$ & $\begin{array}{l}1571.1 \\
69013 \\
\end{array}$ & $\begin{array}{l}71898 . \\
48811\end{array}$ & $\begin{array}{l}18966 . \\
46982\end{array}$ & $\begin{array}{l}3772.0 \\
33796 \\
\end{array}$ & $\begin{array}{l}8418.8 \\
95548\end{array}$ & $\begin{array}{l}- \\
1674.1 \\
54381\end{array}$ & $\begin{array}{l}- \\
440.44 \\
41056 \\
\end{array}$ \\
\hline 203240 & 689055 & 131739 & $\begin{array}{l}- \\
377288 \\
\end{array}$ & $\begin{array}{l}- \\
357002\end{array}$ & $\begin{array}{l}748506 \\
806.7 \\
\end{array}$ & 327492 & 553790 & $\begin{array}{l}538810 \\
194.7 \\
\end{array}$ & $\begin{array}{l}602763 \\
130.3 \\
\end{array}$ & $\begin{array}{l}102059 \\
9222 \\
\end{array}$ & 992920 & $-\overline{446845}$ & $\begin{array}{l}434678 \\
235.3 \\
\end{array}$ & $\begin{array}{l}734017 \\
891.5 \\
\end{array}$ \\
\hline
\end{tabular}


Prediction of Flexural Strength of Chikoko Pozzolana Blended Cement Concrete using Osadebe's Regression Function, Do Onwuka \& S Sule

\begin{tabular}{|c|c|c|c|c|c|c|c|c|c|c|c|c|c|c|}
\hline$Z_{1}$ & $Z_{2}$ & $Z_{3}$ & $Z_{4}$ & $Z_{5}$ & $Z_{1} Z_{2}$ & $Z_{1} Z_{3}$ & $Z_{1} Z_{4}$ & $Z_{1} Z_{5}$ & $Z_{2} Z_{3}$ & $Z_{2} Z_{4}$ & $Z_{2} Z_{5}$ & $Z_{3} Z_{4}$ & $Z_{3} Z_{5}$ & $Z_{4} Z_{5}$ \\
\hline 288.4 & 971.3 & 940.8 & 229.5 & 065.1 & & 090 & 774.7 & & & & 807.9 & 312.9 & & \\
\hline $\begin{array}{l} \\
512711 \\
88.03 \\
\end{array}$ & $\begin{array}{l} \\
169136 \\
856.3\end{array}$ & $\begin{array}{l} \\
338324 \\
33.28 \\
\end{array}$ & $\begin{array}{l}- \\
903654 \\
61.89 \\
\end{array}$ & $\begin{array}{l} \\
900907 \\
32.69 \\
\end{array}$ & $\begin{array}{l}186310 \\
297.8 \\
\end{array}$ & $\begin{array}{l} \\
834610 \\
08.03\end{array}$ & $\begin{array}{l} \\
136266 \\
415.1 \\
\end{array}$ & $\begin{array}{l}135965 \\
529.8 \\
\end{array}$ & $\begin{array}{l}151391 \\
981.8 \\
\end{array}$ & $\begin{array}{l}247498 \\
020.8 \\
\end{array}$ & $\begin{array}{l} \\
246934 \\
067.8 \\
\end{array}$ & $\begin{array}{l} \\
110946 \\
960.6\end{array}$ & $\begin{array}{l}110681 \\
745.3 \\
\end{array}$ & $\begin{array}{l}180457 \\
548.1 \\
\end{array}$ \\
\hline $\begin{array}{l}199870 \\
727.7\end{array}$ & $\begin{array}{l}674187 \\
363.6\end{array}$ & $\begin{array}{l}- \\
128859 \\
058.8\end{array}$ & $\begin{array}{l}- \\
375996 \\
740.6\end{array}$ & $\begin{array}{l}- \\
357252 \\
199.3\end{array}$ & $\begin{array}{l}734223 \\
502.7 \\
\end{array}$ & $\begin{array}{l}- \\
321194 \\
025\end{array}$ & $\begin{array}{l}- \\
548239 \\
088.4\end{array}$ & $\begin{array}{l}534511 \\
271.4 \\
\end{array}$ & $\begin{array}{l}589668 \\
434.2 \\
\end{array}$ & $\begin{array}{l}100779 \\
9342\end{array}$ & $\begin{array}{l}982494 \\
737.7\end{array}$ & $\begin{array}{l}441174 \\
252.8 \\
\end{array}$ & $\begin{array}{l}430048 \\
691.7 \\
\end{array}$ & $\begin{array}{l}733016 \\
952\end{array}$ \\
\hline $\begin{array}{l}- \\
529847 \\
52.73\end{array}$ & $\begin{array}{l}- \\
176624 \\
633.7\end{array}$ & $\begin{array}{l}- \\
353163 \\
62.06\end{array}$ & $\begin{array}{l}- \\
909991 \\
66.45\end{array}$ & $\begin{array}{l}- \\
899651 \\
44.08\end{array}$ & $\begin{array}{l}193544 \\
722.9\end{array}$ & $\begin{array}{l}- \\
866835 \\
96.57\end{array}$ & $\begin{array}{l}- \\
139008 \\
672.6\end{array}$ & $\begin{array}{l}138121 \\
671.5\end{array}$ & $\begin{array}{l}158062 \\
400.8\end{array}$ & $\begin{array}{l}253803 \\
262.2\end{array}$ & $\begin{array}{l}- \\
252165 \\
936.5\end{array}$ & $\begin{array}{l}- \\
113749 \\
553.5\end{array}$ & $\begin{array}{l}113003 \\
066.9\end{array}$ & $\begin{array}{l}180962 \\
694.1\end{array}$ \\
\hline $\begin{array}{l}- \\
458671 \\
948.1\end{array}$ & $\begin{array}{l}- \\
154096 \\
5914\end{array}$ & $\begin{array}{l}- \\
299095 \\
177.5\end{array}$ & $\begin{array}{l}- \\
836943 \\
994.3\end{array}$ & $\begin{array}{l}- \\
805771 \\
395.7\end{array}$ & $\begin{array}{l}168170 \\
9131\end{array}$ & $\begin{array}{l}- \\
741609 \\
265.6\end{array}$ & $\begin{array}{l}- \\
123951 \\
4785\end{array}$ & $\begin{array}{l}121610 \\
6039\end{array}$ & $\begin{array}{l}135836 \\
1013\end{array}$ & $\begin{array}{l}227329 \\
3047\end{array}$ & $\begin{array}{l}- \\
223020 \\
3273\end{array}$ & $\begin{array}{l}- \\
100317 \\
4771\end{array}$ & $\begin{array}{l}984049 \\
480.1\end{array}$ & $\begin{array}{l}164243 \\
1815\end{array}$ \\
\hline
\end{tabular}

Table 5: Flexural Strength Test Results

\begin{tabular}{|c|c|c|c|c|c|c|c|}
\hline Exp. No & Replicates & $\operatorname{Mass}(\mathrm{Kg})$ & Density $\left(\mathrm{Kg} / \mathrm{m}^{3}\right)$ & $\begin{array}{c}\text { Average Density } \rho \\
\left(\mathrm{kg} / \mathrm{m}^{3}\right)\end{array}$ & $\begin{array}{l}\text { Load at Failure } \\
(\mathrm{KN})\end{array}$ & Flexural Strength & $\begin{array}{l}\text { Average Flexural } \\
\text { Strength }\end{array}$ \\
\hline \multirow{3}{*}{1} & A & 27.8 & 2471.111 & & 39 & 5.200 & \\
\hline & $B$ & 26.9 & 2391.111 & 2432.593 & 36 & 4.800 & 4.933 \\
\hline & $\mathrm{C}$ & 27.4 & 2435.556 & & 36 & 4.800 & \\
\hline \multirow{3}{*}{2} & $\mathrm{~A}$ & 28.2 & 2506.667 & & 30 & 4.000 & \\
\hline & $B$ & 27 & 2400.000 & 2447.407 & 22 & 2.933 & 3.644 \\
\hline & C & 27.4 & 2435.556 & & 30 & 4.000 & \\
\hline \multirow{3}{*}{3} & A & 27.1 & 2408.889 & & 28 & 3.733 & \\
\hline & B & 27.1 & 2408.889 & 2388.148 & 20 & 2.667 & 3.467 \\
\hline & C & 26.4 & 2346.667 & & 30 & 4.000 & \\
\hline \multirow{3}{*}{4} & $\mathrm{~A}$ & 26.8 & 2382.222 & & 21 & 2.800 & \\
\hline & B & 27.1 & 2408.889 & 2429.630 & 22 & 2.933 & 2.711 \\
\hline & $\mathrm{C}$ & 28.1 & 2497.778 & & 18 & 2.400 & \\
\hline \multirow{3}{*}{5} & A & 27.8 & 2471.111 & & 26 & 3.467 & \\
\hline & $B$ & 28 & 2488.889 & 2477.037 & 21 & 2.800 & 3.422 \\
\hline & C & 27.8 & 2471.111 & & 30 & 4.000 & \\
\hline \multirow{3}{*}{6} & A & 28.2 & 2506.667 & & 32 & 4.267 & \\
\hline & $B$ & 27.9 & 2480.000 & 2450.370 & 40 & 5.333 & 4.267 \\
\hline & $C$ & 26.6 & 2364.444 & & 24 & 3.200 & \\
\hline \multirow{3}{*}{7} & A & 28.4 & 2524.444 & & 36 & 4.800 & \\
\hline & $B$ & 26.2 & 2328.889 & 2447.407 & 30 & 4.000 & 4.533 \\
\hline & C & 28 & 2488.889 & & 36 & 4.800 & \\
\hline \multirow{3}{*}{8} & A & 28.2 & 2506.667 & & 36 & 4.800 & \\
\hline & $B$ & 28 & 2488.889 & 2465.185 & 24 & 3.200 & 3.733 \\
\hline & $\mathrm{C}$ & 27 & 2400.000 & & 24 & 3.200 & \\
\hline \multirow{3}{*}{9} & A & 28.1 & 2497.778 & & 37 & 4.933 & \\
\hline & B & 27 & 2400.000 & 2432.593 & 20.5 & 2.733 & 4.022 \\
\hline & $C$ & 27 & 2400.000 & & 33 & 4.400 & \\
\hline \multirow{3}{*}{10} & A & 27.1 & 2408.889 & & 35 & 4.667 & \\
\hline & $B$ & 27.2 & 2417.778 & 2420.741 & 19.5 & 2.600 & 3.844 \\
\hline & $\mathrm{C}$ & 27.4 & 2435.556 & & 32 & 4.267 & \\
\hline \multirow{3}{*}{11} & $\mathrm{~A}$ & 28.2 & 2506.667 & & 24 & 3.200 & \\
\hline & $B$ & 27.2 & 2417.778 & 2435.556 & 18.5 & 2.467 & 3.356 \\
\hline & C & 26.8 & 2382.222 & & 33 & 4.400 & \\
\hline \multirow{3}{*}{12} & A & 28 & 2488.889 & & 28 & 3.733 & \\
\hline & $B$ & 28 & 2488.889 & 2459.259 & 22.7 & 3.027 & 3.542 \\
\hline & $\mathrm{C}$ & 27 & 2400.000 & & 29 & 3.867 & \\
\hline \multirow{3}{*}{13} & $\mathrm{~A}$ & 27 & 2400.000 & & 30 & 4.000 & \\
\hline & B & 26.9 & 2391.111 & 2420.741 & 22.5 & 3.000 & 3.311 \\
\hline & C & 27.8 & 2471.111 & & 22 & 2.933 & \\
\hline \multirow{3}{*}{14} & A & 27.9 & 2480.000 & & 34.5 & 4.600 & \\
\hline & B & 27.4 & 2435.556 & 2438.519 & 25 & 3.333 & 3.756 \\
\hline & C & 27 & 2400.000 & & 25 & 3.333 & \\
\hline \multirow{3}{*}{15} & A & 28.2 & 2506.667 & & 29 & 3.867 & \\
\hline & $B$ & 27 & 2400.000 & 2435.556 & 19 & 2.533 & 2.978 \\
\hline & C & 27 & 2400.000 & & 19 & 2.533 & \\
\hline \multicolumn{8}{|c|}{ Control points } \\
\hline \multirow{2}{*}{ C1 } & A & 27.6 & 2453.333 & & 28 & 3.733 & \\
\hline & B & 27.2 & 2417.778 & 2438.519 & 40 & 5.333 & 4.267 \\
\hline
\end{tabular}


Prediction of Flexural Strength of Chikoko Pozzolana Blended Cement Concrete using Osadebe's Regression Function, Do Onwuka \& S Sule

\begin{tabular}{|c|c|c|c|c|c|c|c|}
\hline Exp. No & Replicates & $\operatorname{Mass}(\mathrm{Kg})$ & Density $\left(\mathrm{Kg} / \mathrm{m}^{3}\right)$ & $\begin{array}{c}\text { Average Density } \rho \\
\left(\mathrm{kg} / \mathrm{m}^{3}\right)\end{array}$ & $\begin{array}{l}\text { Load at Failure } \\
(\mathrm{KN})\end{array}$ & Flexural Strength & $\begin{array}{c}\text { Average Flexural } \\
\text { Strength }\end{array}$ \\
\hline & C & 27.5 & 2444.444 & & 28 & 3.733 & \\
\hline \multirow{3}{*}{$\mathrm{C} 2$} & A & 28 & 2488.889 & & 30 & 4.000 & \\
\hline & B & 27 & 2400.000 & 2432.593 & 26 & 3.467 & 3.867 \\
\hline & $C$ & 27.1 & 2408.889 & & 31 & 4.133 & \\
\hline \multirow{3}{*}{ C3 } & A & 27 & 2400.000 & & 32 & 4.267 & \\
\hline & $B$ & 26.8 & 2382.222 & 2402.963 & 21 & 2.800 & 3.689 \\
\hline & $C$ & 27.3 & 2426.667 & & 30 & 4.000 & \\
\hline \multirow{3}{*}{ C4 } & A & 28 & 2488.889 & & 30 & 4.000 & \\
\hline & B & 26.4 & 2346.667 & 2411.852 & 29 & 3.867 & 3.867 \\
\hline & C & 27 & 2400.000 & & 28 & 3.733 & \\
\hline \multirow{3}{*}{ C5 } & A & 27.4 & 2435.556 & & 32 & 4.267 & \\
\hline & B & 28 & 2488.889 & 2447.407 & 21 & 2.800 & 3.689 \\
\hline & C & 27.2 & 2417.778 & & 30 & 4.000 & \\
\hline \multirow{3}{*}{ C6 } & A & 28.1 & 2497.778 & & 28 & 3.733 & \\
\hline & B & 26.6 & 2364.444 & 2429.630 & 28 & 3.733 & 4.178 \\
\hline & C & 27.3 & 2426.667 & & 38 & 5.067 & \\
\hline \multirow{3}{*}{$\mathrm{C7}$} & A & 26.4 & 2346.667 & & 33 & 4.400 & \\
\hline & $B$ & 27.5 & 2444.444 & 2426.667 & 32 & 4.267 & 4.489 \\
\hline & C & 28 & 2488.889 & & 36 & 4.800 & \\
\hline \multirow{3}{*}{ C8 } & A & 27.2 & 2417.778 & & 30 & 4.000 & \\
\hline & $B$ & 28 & 2488.889 & 2438.519 & 34 & 4.533 & 4.178 \\
\hline & $C$ & 27.1 & 2408.889 & & 30 & 4.000 & \\
\hline \multirow{3}{*}{ C9 } & A & 26.3 & 2337.778 & & 30 & 4.000 & \\
\hline & B & 28 & 2488.889 & 2414.815 & 28 & 3.733 & 3.911 \\
\hline & $C$ & 27.2 & 2417.778 & & 30 & 4.000 & \\
\hline \multirow{3}{*}{ C10 } & A & 27.3 & 2426.667 & & 30 & 4.000 & \\
\hline & $B$ & 27 & 2400.000 & 2420.741 & 27 & 3.600 & 3.822 \\
\hline & $\mathrm{C}$ & 27.4 & 2435.556 & & 29 & 3.867 & \\
\hline \multirow{3}{*}{ C11 } & A & 28 & 2488.889 & & 29 & 3.867 & \\
\hline & B & 27 & 2400.000 & 2417.778 & 25 & 3.333 & 3.911 \\
\hline & C & 26.6 & 2364.444 & & 34 & 4.533 & \\
\hline \multirow{3}{*}{$\mathrm{C} 12$} & A & 26.7 & 2373.333 & & 24 & 3.200 & \\
\hline & $B$ & 27.5 & 2444.444 & 2408.889 & 31 & 4.133 & 3.778 \\
\hline & $C$ & 27.1 & 2408.889 & & 26 & 3.467 & \\
\hline \multirow{3}{*}{$\mathrm{C} 13$} & A & 27.1 & 2408.889 & & 25 & 3.333 & \\
\hline & $B$ & 26.9 & 2391.111 & 2414.815 & 34 & 4.533 & 4.133 \\
\hline & $\mathrm{C}$ & 27.5 & 2444.444 & & 34 & 4.533 & \\
\hline \multirow{3}{*}{ C14 } & $\mathrm{A}$ & 27.8 & 2471.111 & & 31 & 4.133 & \\
\hline & B & 27.1 & 2408.889 & 2426.667 & 23 & 3.067 & 3.911 \\
\hline & $\mathrm{C}$ & 27 & 2400.000 & & 34 & 4.533 & \\
\hline \multirow{3}{*}{$\mathrm{C} 15$} & $\mathrm{~A}$ & 26.5 & 2355.556 & & 28 & 3.733 & \\
\hline & B & 27.2 & 2417.778 & 2400.000 & 30 & 4.000 & 3.867 \\
\hline & $c$ & 27.3 & 2426.667 & & 29 & 3.867 & \\
\hline
\end{tabular}

\subsection{The Regression Model}

Substituting the values of $y^{(n)}$ from the test results shown in Table 4 into Equation (33) yields Osadebe's coefficients values as follows: 


$$
\begin{aligned}
\alpha_{1}=10129.60157, & \alpha_{2}-2969.72244, \quad \alpha_{3}=2970.80494, \quad \alpha_{4}=157608673.5, \quad \alpha_{5}=-39230199.22, \quad \alpha_{6} \\
= & -21919.30298, \quad \alpha_{7}=-19274.41211, \quad \alpha_{8}=158913915.9, \quad \alpha_{9}=38562608.66, \quad \alpha_{10} \\
= & 11309.67354, \quad \alpha_{11}=158693269.8, \quad \alpha_{12}=38695844.25, \quad \alpha_{13}=155210497.7, \quad \alpha_{14} \\
= & 40434855.8, \quad \alpha_{15}=354102810.9
\end{aligned}
$$

Substituting the obtained coefficients into Equation (27) yields:

$$
\begin{aligned}
& y=10129.60157 z_{1},-2969.72244 z_{2}-2970.80494 z_{3}+157608673 . z_{4}-39230199.22 z_{5}, \\
& -21919.30298 z_{1} z_{2}-19274.41211 z_{1} z_{3}+158913915.9 z_{1} z_{4}+38562608.66 z_{1} z_{5} \\
& +11309.67354 z_{2} z_{3}+158693269.8 Z z_{4}+38695844.25 z \quad z_{5}+155210497.7 z \quad z_{4} \\
& +40434855.8 Z \quad Z_{5} \quad 354102810.9 Z \quad Z_{5}
\end{aligned}
$$

Equation (36) is the required regression model for the prediction and optimization of flexural strength of chikokocement concrete based on Osadebe's second degree polynomial.

\subsection{Test of Adequacy of the Model}

Equation (36) was tested for adequacy using Fisher test in Table 5 and was found to be adequate.

Table 5: Fisher test for control points

\begin{tabular}{ccccccc}
\hline Control points & $\mathrm{y}_{\mathrm{o}}$ & $\mathrm{yp}_{\mathrm{p}}$ & $y_{o}-\bar{y}_{o}$ & $y_{p}-\bar{y}_{p}$ & $\left(y_{o}-\bar{y}_{o}\right)^{2}$ & $\left(y_{p}-\bar{y}_{p}\right)^{2}$ \\
\hline C1 & 4.267 & 4.276 & 0.297 & 0.341 & 0.088 & 0.116 \\
C2 & 3.867 & 3.901 & -0.103 & -0.034 & 0.011 & 0.001 \\
C3 & 3.689 & 3.541 & -0.281 & -0.394 & 0.079 & 0.155 \\
C4 & 3.867 & 3.910 & -0.103 & -0.025 & 0.011 & 0.001 \\
C5 & 3.689 & 3.754 & -0.281 & -0.181 & 0.079 & 0.033 \\
C6 & 4.178 & 4.048 & 0.208 & 0.113 & 0.043 & 0.013 \\
C7 & 4.489 & 4.465 & 0.519 & 0.530 & 0.269 & 0.281 \\
C8 & 4.178 & 3.965 & 0.208 & 0.030 & 0.043 & 0.001 \\
C9 & 3.911 & 4.111 & -0.059 & 0.176 & 0.004 & 0.031 \\
C10 & 3.822 & 3.783 & -0.148 & -0.152 & 0.022 & 0.023 \\
C11 & 3.911 & 3.881 & -0.059 & -0.054 & 0.004 & 0.003 \\
C12 & 3.778 & 3.577 & -0.192 & -0.358 & 0.037 & 0.128 \\
C13 & 4.133 & 4.165 & 0.163 & 0.230 & 0.026 & 0.053 \\
C14 & 3.911 & 3.810 & -0.059 & -0.125 & 0.004 & 0.016 \\
C15 & 3.867 & 3.838 & -0.103 & -0.097 & 0.011 & 0.009 \\
$\Sigma$ & 3.970 & 3.935 & & $\Sigma$ & 0.730 & 0.864 \\
\hline
\end{tabular}

Legend:

$$
\bar{y}_{o}=\frac{\sum y_{o}}{n} ; \bar{y}_{p}=\frac{\sum y_{p}}{n}
$$

The F- statistics is the ratio of sample variances and is given by:

$$
F=S_{1}{ }^{2} / S_{2}{ }^{2}
$$

where: $S_{1}{ }^{2}$ is always the larger value of the sample variances

Let $S_{o}{ }^{2}, S_{p}{ }^{2}=$ variance of observed and predicted data respectively

Then,

$$
S_{o}{ }^{2}=\frac{0.730}{14} 0.05212, \quad S_{p}{ }^{2}=\frac{0.864}{14}=0.06169
$$

If $S_{o}{ }^{2}=S_{p}{ }^{2}$ it implies that the variances are the equal at all experimental points. $S_{o}{ }^{2}$ not being equal to $S_{p}{ }^{2}$ shows that the population variances are not the same at all experimental points. The acceptance of Null hypothesis implies that the difference between the sample variances, $S_{o}{ }^{2}$ and $S_{p}{ }^{2}$ at $5 \%$ level of significance is not significant by applying the Fisher test.

$$
F=\frac{0.06169}{0.05212}=1.1838
$$

From Fisher table, $F_{0.95}(14,14)=2.48[17]$.

The calculated value of $\mathrm{F}$ is less than the value from

Fisher table. Hence the model is adequate.

\section{CONCLUSION}

The experimental data are well fitted into the predictive and optimization model showing the effectiveness of Osadebe's model in the prediction and optimization of concrete flexural strength of chikoko pozzolana blended cement concrete. The strength of concrete depends on 
the proportions of the component materials: water, cement, chikoko, sand and coarse aggregates. The results of the Fisher test showed that the predictive and optimization model is adequate.

The model can be used to predict and optimize other structural properties of chikoko pozzolana blended cement concrete. With the formulated model, any desired value of flexural strength, given any mix ratios can be easily determined and can also determine the mix ratios when a desired value of flexural strength is given.

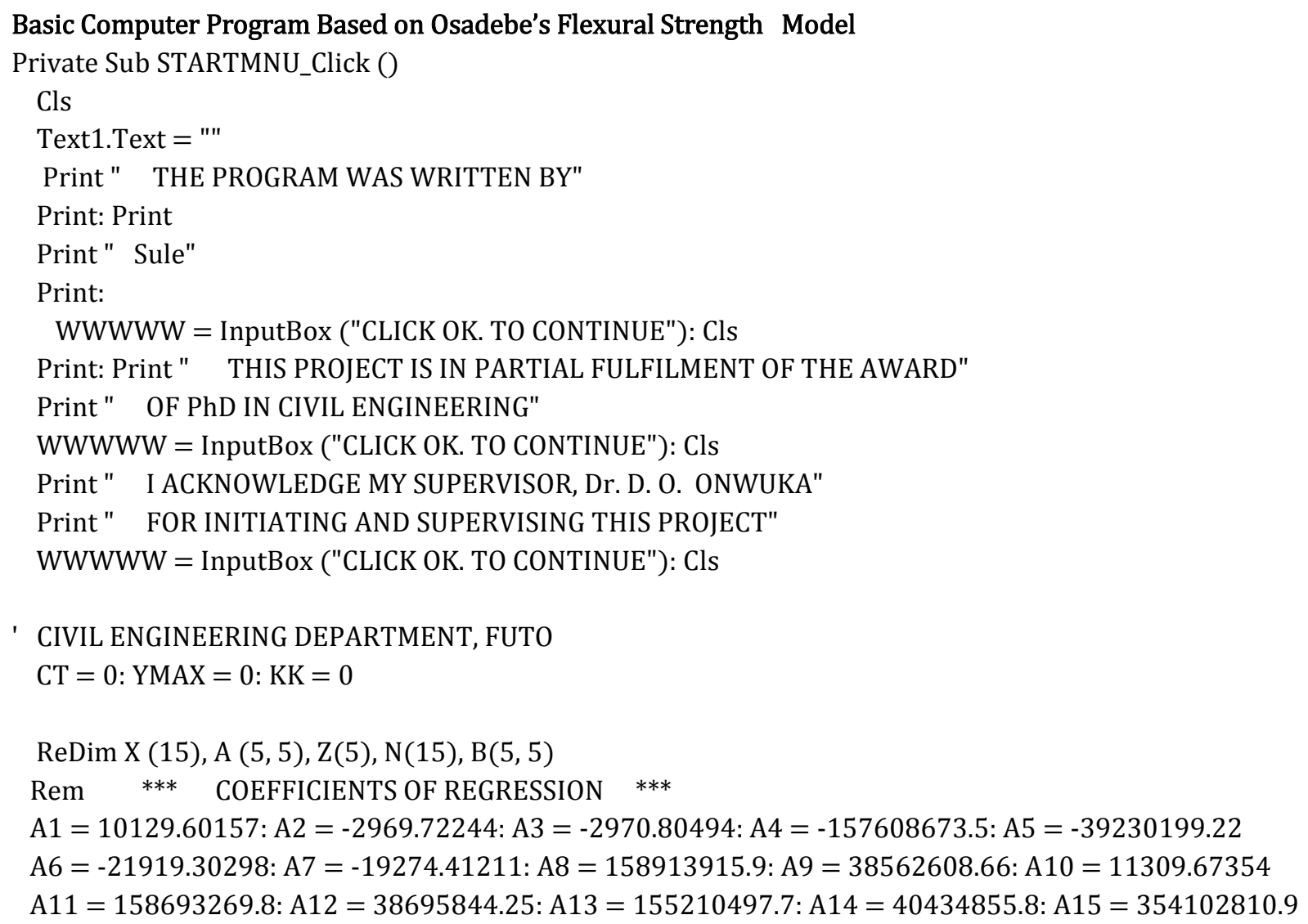


$\mathrm{Z} 23=\mathrm{Z} 2+\mathrm{Z3}$

Rem CACCULATING ACTUAL STRENGTH

$\mathrm{YACT}=\mathrm{A} 1 * \mathrm{Z} 1+\mathrm{A} 2 * \mathrm{Z} 2+\mathrm{A} 3 * \mathrm{Z} 3+\mathrm{A} 4 * \mathrm{Z} 4+\mathrm{A} 5 * \mathrm{Z} 5$

$\mathrm{YACT}=\mathrm{YACT}+\mathrm{A} 6 * \mathrm{Z} 6+\mathrm{A} 7 * \mathrm{Z} 7+\mathrm{A} 8 * \mathrm{Z} 8+\mathrm{A} 9 * \mathrm{Z} 9+\mathrm{A} 10 * \mathrm{Z} 10$

$\mathrm{YACT}=\mathrm{YACT}+\mathrm{A} 11 * \mathrm{Z} 11+\mathrm{A} 12 * \mathrm{Z} 12+\mathrm{A} 13 * \mathrm{Z} 13+\mathrm{A} 14 * \mathrm{Z} 14+\mathrm{A} 15 * \mathrm{Z} 15$

$\mathrm{Y}=\mathrm{YACT}$

If Z1 / Z23 $<0.52$ Then GoTo 30

If $\mathrm{Z} 1+\mathrm{Z} 2+\mathrm{Z} 3+\mathrm{Z} 4+\mathrm{Z} 5<>1$ Then GoTo 30 'or $\mathrm{Z} 1+\mathrm{Z} 2+\mathrm{Z} 3+\mathrm{Z} 4+\mathrm{Z} 5<1$

If $Y>Y Y-0.05$ and $Y<Y Y+0.05$ Then GoTo 20 Else GoTo 30

20 Text1.Text $=$ Text1.Text + CStr ("flexural Strength" \& vbTab \& Format (YACT, "0.00\#") \& ",") \& vbTab Text1.Text $=$ Text1.Text + CStr (" WATER =" \& vbTab \& Format $(Z 1 /$ Z23, "0.00\#") \& ",") \& vbTab Text1.Text $=$ Text1.Text + CStr (" CEMENT $="$ \& vbTab \& Format $(Z 2 / Z 23$, "0.00\#") \& ",") \& vbTab Text1.Text $=$ Text1.Text + CStr (" ASH =" \& vbTab \& Format (Z3 / Z23, "0.00\#") \& ",") \& vbTab Text1.Text $=$ Text1.Text + CStr (" SAND $="$ \& vbTab \& Format $(Z 4 / Z 23$, "0.00\#") \& ",") \& vbTab Text1.Text $=$ Text1.Text + CStr $(" \quad$ COARSE AGG $=" \&$ vbTab \&Format $(Z 5 /$ Z23, "0.00\#")) \& vbCrLf

30

Next Z4

Next Z3

Next Z2

Next Z1

70 'Print "Sorry! Desired strength is outside the range of the model" 111 GoTo 222

100 Rem $^{* * *}$ Here is where the INPUT of the Principal Predictors will be made ${ }^{* * *}$ Cls

$\mathrm{Z} 1$ = InputBox ("What is Water/Cement ratio"): $\mathrm{Z} 1=\mathrm{Z} 1 * 1$

$\mathrm{Z} 2=$ InputBox ("What is Cement value"): $\mathrm{Z} 2=\mathrm{Z} 2 * 1$

$\mathrm{Z3}=$ InputBox ("What is Ash value"): $\mathrm{Z} 3=\mathrm{Z3} * 1$

$\mathrm{Z} 4=$ InputBox ("What is Sand value"): $\mathrm{Z} 4=\mathrm{Z} 4 * 1$

$\mathrm{Z} 5$ = InputBox ("What is Coarse Agg value"): Z5 = Z5 * 1

$\mathrm{Z} 23=\mathrm{Z} 2+\mathrm{Z} 3$

$\mathrm{TZT}=\mathrm{Z} 1+\mathrm{Z} 2+\mathrm{Z} 3+\mathrm{Z} 4+\mathrm{Z} 5$

$\mathrm{Z} 1=\mathrm{Z1} / \mathrm{TZT}: \mathrm{Z2}=\mathrm{Z2} / \mathrm{TZT}: \mathrm{Z3}=\mathrm{Z3} / \mathrm{TZT}$

$\mathrm{Z} 4=\mathrm{Z} 4 / \mathrm{TZT}: \mathrm{Z5}=\mathrm{Z} 5 / \mathrm{TZT}$

Rem *** The Binary Predictors will be calculated here ${ }^{* * *}$

$\mathrm{Z} 6=\mathrm{Z} 1 * \mathrm{Z} 2: \mathrm{Z} 7=\mathrm{Z} 1 * \mathrm{Z} 3: \mathrm{Z} 8=\mathrm{Z} 1 * \mathrm{Z} 4: \mathrm{Z} 9=\mathrm{Z} 1 * \mathrm{Z} 5: \mathrm{Z} 10=\mathrm{Z} 2 * \mathrm{Z} 3$

$\mathrm{Z} 11=\mathrm{Z} 2 * \mathrm{Z} 4: \quad \mathrm{Z} 12=\mathrm{Z} 2 * \mathrm{Z} 5: \mathrm{Z} 13=\mathrm{Z} 3 * \mathrm{Z} 4: \mathrm{Z} 14=\mathrm{Z} 3 * \mathrm{Z} 5: \mathrm{Z} 15=\mathrm{Z} 4 * \mathrm{Z} 5$

Rem CACCULATING ACTUAL STRENGTH

$\mathrm{YACT}=\mathrm{A} 1 * \mathrm{Z} 1+\mathrm{A} 2 * \mathrm{Z} 2+\mathrm{A} 3 * \mathrm{Z} 3+\mathrm{A} 4 * \mathrm{Z} 4+\mathrm{A} 5 * \mathrm{Z} 5$

$\mathrm{YACT}=\mathrm{YACT}+\mathrm{A} 6 * \mathrm{Z} 6+\mathrm{A} 7 * \mathrm{Z} 7+\mathrm{A} 8 * \mathrm{Z} 8+\mathrm{A} 9 * \mathrm{Z} 9+\mathrm{A} 10 * \mathrm{Z} 10$

$\mathrm{YACT}=\mathrm{YACT}+\mathrm{A} 11 * \mathrm{Z} 11+\mathrm{A} 12 * \mathrm{Z} 12+\mathrm{A} 13 * \mathrm{Z} 13+\mathrm{A} 14 * \mathrm{Z} 14+\mathrm{A} 15 * \mathrm{Z} 15$

Text1.Text = Text1.Text + CStr ("FLEXURAL Strength" \& vbTab \& Format (YACT, "0.00\#") \& ",") \& vbTab Text1.Text $=$ Text1.Text + CStr (" WATER =" \& vbTab \& Format $(\mathrm{Z1} / \mathrm{Z23}$, "0.00\#") \& ",") \& vbTab Text1.Text $=$ Text1.Text + CStr (" CEMENT $="$ \& vbTab \& Format $(Z 2 /$ Z23, "0.00\#") \& ",") \& vbTab Text1.Text $=$ Text1.Text + CStr (" ASH =" \& vbTab \& Format(Z3 / Z23, "0.00\#") \& ",") \& vbTab Text1.Text $=$ Text1.Text $+\operatorname{CStr}(" \quad$ Sand $="$ \& vbTab \& Format $(Z 4 /$ Z23, "0.00\#") \& ",") \& vbCrLf 
Text1.Text $=$ Text1.Text + CStr(" $\quad$ COARSE AGG =" \& vbTab \& Format(Z5 / Z23, "0.00\#") \& ",") \& vbCrLf 222

End Sub

Private Sub STOPMNU_Click ()

End

End Sub

WHAT IS THE DESIRED FLEXURAL STRENGHT? $4.255 / \mathrm{mm}^{2}$

Flexural Strength $\quad 4.255$, WATER $=0.521$

$=2.123, \quad$ COARSE AGG $=\quad 4.249$

Flexural Strength $4.277, \quad$ WATER $=0.522$,

$=2.123, \quad$ COARSE AGG $=\quad 4.247$

Flexural Strength 4.232 , WATER $=0.524$,

$=\quad 2.122, \quad$ COARSE AGG $=\quad 4.246$

Flexural Strength $4.263, \quad$ WATER $=0.522$,

$=\quad 2.103, \quad$ COARSE AGG $=\quad 4.207$

Flexural Strength 4.254 , WATER $=0.526$,

$=\quad 2.121, \quad$ COARSE AGG $=\quad 4.245$

Flexural Strength $4.234, \quad$ WATER $=0.526$,

$=\quad 2.122, \quad$ COARSE AGG $=\quad 4.244$

Flexural Strength 4.211, WATER =

$=\quad 2.121, \quad$ COARSE AGG $=\quad 4.245$

Flexural strength result (for a given mix ratio)
$\mathrm{Y}=$
4.589, WATER $=0.53$,
2.00, COARSE AGG. $=4.00$
CEMENT
0.94
$\mathrm{ASH}=$
0.06
SAND $=$

\section{REFERENCES}

[1] Onwuka, D. O., Anyaogu, L., Chijioke, C., and Okoye, P. C."Prediction and Optimization of Compressive Strength of Sawdust Ash-Cement Concrete Using Scheffe's Simplex Design", International Journal of Scientific and Research Publications, Vol. 3, pp1-8. 2013.

[2] Anyaogu, L. and Ezeh, J. C. "Optimization of Compressive Strength of Fly Ash Blended Cement Concrete using Scheffe's Simplex Theory", Natural and Applied Sciences, Vol. 4, No.2, pp. 177-186. 2013.

[3] Obam, S. O. and Osadebe, N. N. "Optimization of Compressive Strength of Rice Husk Ash Pozzolan Concrete", Journal of Scientific and Industrial Studies, 1(2), pp51-57. 2006.

[4] Neville, A. M."Properties of Concrete", Longman Ltd., England, 1996.

[5] Manasseh, J. "A Review of Partial Replacement with Some Agro Wastes", Nigerian Journal of Technology, 29(2), pp. 12-20. 2010.

[6] Osadebe, N. N. and Obam, S. O."Improvement in the Properties of Concrete by Partial Substitution of Ordinary Portland Cement Rice Husk Ash", Proceedings of Annual Conference of IRDI Science

and Technology Forum, University of Nigeria Nsukka. 2(2), pp 87-91. 2006.

[7] Ndububa, E. E. and Osadebe, N. N. "An Optimization of the Flexural Strength of Fibre Cement Mixture Using Scheffe's Simplex Lattice", NSE Technical Transaction, 42, (1), ,pp 1-17. 2007.

[8] Osadebe, N. N. and Nwakonobi, T. U. "Structural Characteristics of Laterized Concrete at Optimum Mix Proportion", Nigeria Journal of Technology, 34(1), pp 12-17. 2007.

[9] Osadebe, N. N., Mbajiorgu, C. C. and Nwkonobi, T. U. "An Optimization Model for Laterized Concrete Mix Proportioning in Building Constructions", Nigerian Journal of Technology, 26(1), 2007, pp 37-46.

[10] Otoko, G. R. "On The Economic Use of Cement in Soil Stabilization", International Journal of Engineering and Technology Research, Vol2. No.1, pp 01-07. 2014.

[11] Otoko, G. R. and Chinwah, J. G. "The use of Garri as Admixture in Hot Weather Concreting", The Journal of Nigerian Institute of Structural Engineers, Vol.1, No.4, pp 13-18. 1991.

[12] Emesiobi, F. C. "Further Use of Chikoko Mud Pozzolana for Economical Sandcrete Blocks 
Production", NSE Technical Transaction, 39(3), pp 16-23. 2004,

[13] Kovacs, R. "Effect of Hydration Products on the Properties of Fly-Ash Concrete", Cement and concrete research, 5, No.1, pp. 73-82. 1975.

[14] Obam, S. O. "A Mathematical Model for Optimization of Strength of Concrete: A case study for shear modulus of Rice Husk Ash Concrete", Journal of Industrial Engineering International, Vol. 5, No.9, pp 76-84. 2009.
[15] BS 812."Specification for Portland cement", British Standards Institution, 1978.

[16] BS 812: Part 1. "Methods for Determination of Particle Size and Shape", 1975.

[17] Vining, Geoffery G. "Statistical Methods for Engineers", Brook/Cole publishing Company, USA, 1997. 